\title{
Trajectory Design Using the Center Manifold Theory in the Circular Restricted Three-Body Problem
}

\author{
By Yuki AKIYAmA, ${ }^{1)}$ Mai BANDO, ${ }^{1)}$ Hamidreza NematI ${ }^{1)}$ and Shinji HоKAмото ${ }^{1)}$ \\ ${ }^{1)}$ Department of Aeronautics and Astronautics, Kyushu University, Fukuoka, Japan
}

(Received July 31st, 2015)

\begin{abstract}
Lagrangian points are libration points in the circular restricted three-body problem. Three of them are collinear libration points, and known to have unstable periodic and quasi-periodic orbits in the vicinity of themselves. This study aims to design periodic and quasi-periodic orbits from the center manifold theory standpoint. In this paper, a new approach is proposed to design periodic and quasi-periodic orbits around the collinear libration points based upon the successive approximate method. First, the verification of the proposed approach is discussed by evaluating obtained orbits. Then the relation between the initial condition and the type of resulting orbits is investigated. In conclusion, this paper reveals that the proposed method significantly reduces the effort to obtain bounded orbits in the circular restricted three-body problem. The proposed method needs neither an initial guess for the periodic orbit nor the complex algebraic manipulation unlike the conventional methods designing periodic or quasi-periodic orbits. Moreover, a guide to choose the initial condition to obtain desired orbits is given.
\end{abstract}

Key Words: Center Manifold Theory, Circular Restricted Three-Body Problem, Halo Orbit

$\begin{array}{cll}\text { Nomenclature } & & \\ \boldsymbol{A}, \boldsymbol{B}, \tilde{\boldsymbol{B}} & : & \text { coefficient matrix } \\ \boldsymbol{C}_{i} & : & \text { constant derived by } \sigma_{i}(i=1, \cdots, 5) \\ \boldsymbol{D} & : & \text { block diagonal matrix } \\ \tilde{\boldsymbol{F}}, \boldsymbol{G} & : & \text { nonlinear term } \\ \boldsymbol{f}, g, h & : & \text { nonlinear term in the diagonal system } \\ L_{i} & : & \text { position of Lagrangian point } \\ & & (i=1, \cdots, 5) \\ l_{i} & : & \text { distance of collinear Lagrangian } \\ & & \text { point from the origin }(i=1, \cdots, 3) \\ M_{1}, M_{2} & & \text { mass of body } \\ \boldsymbol{P}, \boldsymbol{Q}, \boldsymbol{R} & : & \text { partitioned matrix of matrix } \boldsymbol{D} \\ r_{1}, r_{2} & : & \text { distance from the center of mass } \\ \boldsymbol{T} & : & \text { transformation matrix } \\ t & : & \text { nominalized time } \\ x, y, z, X, Y, Z & : & \text { position } \\ \boldsymbol{X}, \boldsymbol{Z} & : & \text { state variable } \\ z_{i}^{c}, z^{s}, z^{u} & : & \text { state in the diagonal system }(i=1, \cdots, 4) \\ \Lambda_{i} & : & \text { constant derived by } \sigma_{i} \\ \lambda_{i} & : & \text { eigenvalue of matrix } \boldsymbol{A} \\ \boldsymbol{\xi} & : & \text { initial state of } \boldsymbol{z}^{c} \\ \rho & : & \text { mass ratio } \\ \sigma_{i} & : & \text { constant derived by } \rho \text { and } l_{i} \\ \phi, \psi & : & \text { center manifold } \\ \text { Subscripts } & : & \text { initial } \\ 0 & : & \text { iteration number } \\ k & & \end{array}$

\section{Introduction}

Spacecraft trajectories are influenced by various perturbations including the gravitational field of planets during missions. In the two-body problem, there exist the optimization theories of the trajectory in the sense of minimizing the fuel consumption in the literature. ${ }^{1)}$ For the $n$-body problem, however, optimization theories have not been established yet. The three-body problem is the representative $n$-body problem. The circular restricted three-body problem (CRTBP) supposes that two massive bodies move in the circular orbits around their common center of mass and the third body is much smaller than the other two. For the CRTBP, there are five libration points, called Lagrangian points, which are led from the geometric relationship. ${ }^{2)}$ Among them, the collinear libration points are located in the saddle points of the potential surface. ${ }^{3)}$ Moreover, the existences of several types of bounded orbits in the vicinity of the collinear libration points are known as libration point orbits: Lyapunov, vertical, and halo orbits are periodic and Lissajous and quasi-halo orbits are quasi-periodic. ${ }^{4)-6)}$ In addition, these libration point orbits associate with unstable and stable manifolds. Therefore, it is possible to inject and eject a spacecraft into these orbits by using these manifolds without any energy consumption. In 2001, the trajectory based on dynamical systems theory was applied in the Genesis mission operated by NASA JPL. ${ }^{7)}$ This revealed that fuel efficient trajectories can be designed by using characteristics of the system.

Determination of a reference orbit for a desired mission is a fundamental problem. An accurate initial condition of a libration point orbit is needed to implement various missions around the Lagrangian point, since for ideal case it generates a periodic or quasi-periodic orbit and it is possible to maintain desired orbits without any control for long time. In constructing a control law to realize a desired mission and optimizing a trajectory, analytical methods to design halo orbits are preferable to numerical methods. In computing libration point orbits, the differential correction method is often used. ${ }^{3)}$, 8) However, while it provides a good initial condition, it cannot provide with analytical information which is useful for additional analyses. On the other hand, an analytical method known as the LindstedtPoincaré method provides analytical expression of a libration 
point orbit as functions of time. ${ }^{9),}{ }^{10)}$ However it requires high order expansions to obtain a good initial condition of a libration point orbit. Furthermore, complex algebraic manipulations and further numerical adjustments are necessary to obtain accurate halo orbits.

The main purpose of this study is to design the spacecraft trajectory analytically by exploiting the property of the center manifold theory in the CRTBP. Especially, a new semianalytical theory to approximate a solution to ordinal differential equations on center manifold ${ }^{11)}$ is applied to design libration point orbits around the Lagrangian points. Typical methods to calculate a center manifold based on the Taylor series approximation give rise to several difficulties regarding the operational complexity. In contrast, the analytical approximation theory is a successive iteration method which is easy to implement in computers. Moreover, a semi-analytical approximation method does not require complex operations with high computational costs. In addition, the proposed method is different from the differential correction method and the Lindstedt-Poincaré method. The approach is a semi-analytical method to compute periodic and quasi-periodic orbits and does not require algebraic manipulations for the higher order, while the Lindstedt-Poincaré is not the successive algorithm. Consequently, the approach offers a way to design not only periodic and quasi-periodic orbits but also formation flying or single spacecraft missions. In addition, spacecraft which is on the center manifold can fly around Lagrangian points without any control. Therefore, the proposed method is expected to be useful to realize more fuel efficient missions operated around the Lagrangian points. To verify the proposed approach, the periodic and quasi-periodic orbits obtained by the numerical calculations are shown. Furthermore the dependence between the initial condition and the type of resulting orbits is discussed.

\section{Equations of Motion in CRTBP}

In the CRTBP, the equations of motion in the nondimensional form ${ }^{12)}$ are expressed as

$$
\begin{aligned}
X^{\prime \prime}-2 Y^{\prime}-X & =-\frac{1-\rho}{r_{1}^{3}}(X+\rho)-\frac{\rho}{r_{2}^{3}}(X-1+\rho) \\
Y^{\prime \prime}+2 X^{\prime}-Y & =-\frac{1-\rho}{r_{1}^{3}} Y-\frac{\rho}{r_{2}^{3}} Y \\
Z^{\prime \prime} & =-\frac{1-\rho}{r_{1}^{3}} Z-\frac{\rho}{r_{2}^{3}} Z
\end{aligned}
$$

where $\{X, Y, Z\}$ is the rotating frame whose origin is the barycenter of the system, the coordinates are normalized by the distance between two main bodies and the time by the period of the circular orbit, $\mu=M_{2} /\left(M_{1}+M_{2}\right), M_{1}$ and $M_{2}$ are the masses of the two main bodies with $M_{1}>M_{2}$, and

$$
\begin{aligned}
& r_{1}=\left[(X+\mu)^{2}+Y^{2}+Z^{2}\right]^{1 / 2} \\
& r_{2}=\left[(X-1+\mu)^{2}+Y^{2}+Z^{2}\right]^{1 / 2}
\end{aligned}
$$

Furthermore, the differentiation with respect to the nondimensional time is denoted by '. Equation (1) has stationary points known as Lagrangian points $L_{i}$ satisfying

$$
\begin{aligned}
& X=\frac{1-\mu}{r_{1}^{3}}(X+\mu)+\frac{\mu}{r_{2}^{3}}(X-1+\mu) \\
& Y=\frac{1-\mu}{r_{1}^{3}} Y+\frac{\mu}{r_{2}^{3}} Y \\
& Z=0
\end{aligned}
$$

and

$$
\begin{aligned}
& L_{1}=\left(l_{1}(\mu), 0,0\right), \quad L_{2}=\left(l_{2}(\mu), 0,0\right), \quad L_{3}=\left(l_{3}(\mu), 0,0\right) \\
& L_{4}=(1 / 2-\mu, \sqrt{3} / 2,0), \quad L_{5}=(1 / 2-\mu,-\sqrt{3} / 2,0)
\end{aligned}
$$

where $l_{i}(\mu)$ are determined by setting $Y=0$ and solving the first equation of Eq. (2). ${ }^{1)}$

To describe the motion near a collinear equilibrium point $L_{i}$ ( $i=1,2,3)$, it is convenient to use the coordinate system with the origin at $L_{i}$. Replacing $\{X, Y, Z\}$ by $\left\{x+l_{i}, y, z\right\}$, Eq. (1) can be rewritten as

$$
\begin{aligned}
x^{\prime \prime}-2 y^{\prime}-x & =-\frac{1-\mu}{r_{1}^{3}}\left(x+l_{i}+\mu\right)-\frac{\mu}{r_{2}^{3}}\left(x+l_{i}-1+\mu\right)+l_{i} \\
y^{\prime \prime}+2 x^{\prime}-y & =-\frac{1-\mu}{r_{1}^{3}} y-\frac{\mu}{r_{2}^{3}} y \\
z^{\prime \prime} & =-\frac{1-\mu}{r_{1}^{3}} z-\frac{\mu}{r_{2}^{3}} z
\end{aligned}
$$

where

$$
\begin{aligned}
& r_{1}=\left[\left(x+l_{i}+\rho\right)^{2}+y^{2}+z^{2}\right]^{1 / 2} \\
& r_{2}=\left[\left(x+l_{i}-1+\rho\right)^{2}+y^{2}+z^{2}\right]^{1 / 2}
\end{aligned}
$$

The state space form of Eq. (3) can be represented as

$$
\boldsymbol{X}^{\prime}=\boldsymbol{A} \boldsymbol{X}+\boldsymbol{B} \tilde{\boldsymbol{F}}(\boldsymbol{X})
$$

where $\boldsymbol{X}=\left[x, y, z, x^{\prime}, y^{\prime}, z^{\prime}\right]^{T}$, and

$$
\begin{aligned}
& \boldsymbol{A}=\left[\begin{array}{cccccc}
0 & 0 & 0 & 1 & 0 & 0 \\
0 & 0 & 0 & 0 & 1 & 0 \\
0 & 0 & 0 & 0 & 0 & 1 \\
2 \sigma_{i}+1 & 0 & 0 & 0 & 2 & 0 \\
0 & 1-\sigma_{i} & 0 & -2 & 0 & 0 \\
0 & 0 & -\sigma_{i} & 0 & 0 & 0
\end{array}\right] \\
& \boldsymbol{B}=\left[\begin{array}{lll}
0 & 0 & 0 \\
0 & 0 & 0 \\
0 & 0 & 0 \\
1 & 0 & 0 \\
0 & 1 & 0 \\
0 & 0 & 1
\end{array}\right] \\
& \tilde{\boldsymbol{F}}(\boldsymbol{X})=\left[\begin{array}{c}
-\frac{1-\rho}{r_{1}^{3}}\left(x+l_{i}+\rho\right)-\frac{\rho}{r_{2}^{3}}\left(x+l_{i}-1+\rho\right)+l_{i}-2 \sigma_{i} x \\
-\frac{1-\rho}{r_{1}^{3}} y-\frac{\rho}{r_{2}^{3}} y+\sigma_{i} y \\
-\frac{1-\rho}{r_{1}^{3}} z-\frac{\rho}{r_{2}^{3}} z+\sigma_{i} z
\end{array}\right] \\
& \sigma_{i}=\frac{1-\rho}{\left|l_{i}(\rho)+\rho\right|^{3}}+\frac{\rho}{\left|l_{i}(\rho)-1+\rho\right|^{3}}
\end{aligned}
$$




\section{Strategy of Designing Periodic and Quasi-Periodic Or- bits}

In this section, a new approach of designing periodic and quasi-periodic orbits is proposed. Generally, the state lying on a stable (unstable) manifold converges to (diverges from) the equilibrium point of the dynamical system as time passes. Therefore, only the state on the center manifold can form libration point orbits. To obtain periodic and quasi-periodic orbits, the center manifold and the reduced system lying on the center manifold are calculated by following steps.

\subsection{Deriving diagonal form}

To apply the center manifold theory, the diagonal form of Eq. (4) is calculated. Eigenvalues of matrix $\boldsymbol{A}$ are computed as

$$
\begin{array}{lll}
\lambda_{1}=\Lambda_{1} i, & \lambda_{2}=-\Lambda_{1} i, & \lambda_{3}=\Lambda_{2} i \\
\lambda_{4}=-\Lambda_{2} i, & \lambda_{5}=\Lambda_{3}, & \lambda_{6}=-\Lambda_{3}
\end{array}
$$

where

$$
\begin{aligned}
& \Lambda_{1}=\sqrt{\sigma_{i}} \\
& \Lambda_{2}=\sqrt{\frac{\sqrt{\sigma_{i}\left(9 \sigma_{i}-8\right)}}{2}-\left(\frac{\sigma_{i}}{2}-1\right)} \\
& \Lambda_{3}=\sqrt{\frac{\sqrt{\sigma_{i}\left(9 \sigma_{i}-8\right)}}{2}+\left(\frac{\sigma_{i}}{2}-1\right)}
\end{aligned}
$$

The matrix $\boldsymbol{A}$ has four eigenvalues with zero real parts, one eigenvalue with a negative real number and one eigenvalue with a positive real number. Generally, a matrix $\boldsymbol{A}$ can be transformed into a block diagonal matrix $\boldsymbol{D}$ by a transformation matrix $\boldsymbol{T}$

$$
D=T^{-1} A T=\left[\begin{array}{lll}
P & & 0 \\
& Q & \\
0 & & R
\end{array}\right]
$$

where all eigenvalues of $\boldsymbol{P}$ have zero real parts, all eigenvalues of $\boldsymbol{Q}$ have negative real parts and all eigenvalues of $\boldsymbol{R}$ have positive real parts. In the case of this CRTBP, $Q=-\Lambda_{3}$ and $R=\Lambda_{3}$, and $\boldsymbol{P}$ is expressed as

$$
\boldsymbol{P}=\left[\begin{array}{cccc}
0 & \Lambda_{1} & 0 & 0 \\
-\Lambda_{1} & 0 & 0 & 0 \\
0 & 0 & 0 & \Lambda_{2} \\
0 & 0 & -\Lambda_{2} & 0
\end{array}\right]
$$

The transformation matrix $\boldsymbol{T}$ is expressed as

$$
\boldsymbol{T}=\left[\begin{array}{cccccc}
0 & 0 & -C_{2} & 0 & -C_{1} & -C_{1} \\
0 & 0 & 0 & -C_{3} \Lambda_{3} & -C_{3} \Lambda_{2} & C_{3} \Lambda_{2} \\
0 & -1 / \Lambda_{1} & 0 & 0 & 0 & 0 \\
0 & 0 & 0 & -C_{2} \Lambda_{2} & C_{1} \Lambda_{3} & -C_{1} \Lambda_{3} \\
0 & 0 & 1 & 0 & 1 & 1 \\
1 & 0 & 0 & 0 & 0 & 0
\end{array}\right]
$$

where

$$
\begin{aligned}
& C_{1}=\frac{1}{4\left(2 \sigma_{i}+1\right)}\left[3 \sigma_{i}+4+\sqrt{\sigma_{i}\left(9 \sigma_{i}-8\right)}\right] \\
& C_{2}=\frac{1}{4\left(2 \sigma_{i}+1\right)}\left[3 \sigma_{i}+4-\sqrt{\sigma_{i}\left(9 \sigma_{i}-8\right)}\right] \\
& C_{3}=\frac{1}{\sqrt{\left(2 \sigma_{i}+1\right)\left(\sigma_{i}-1\right)}}
\end{aligned}
$$

Then, new state variable $\boldsymbol{Z}=\boldsymbol{T}^{-1} \boldsymbol{X}$ is given by

$$
\boldsymbol{Z}=\left[\begin{array}{c}
z^{\prime} \\
-\Lambda_{1} z \\
\frac{C_{5}}{C_{4}} x+\frac{C_{1} C_{5}}{C_{4}} y^{\prime} \\
-\frac{C_{1} \Lambda_{3}}{C_{3} C_{4}} y-\frac{\Lambda_{2}}{C_{4}} x^{\prime} \\
-\frac{1}{2}\left(\frac{C_{5}}{C_{4}} x+\frac{C_{2} \Lambda_{2}}{C_{3} C_{4}} y-\frac{\Lambda_{3}}{C_{4}} x^{\prime}+\frac{C_{2} C_{5}}{C_{4}} y^{\prime}\right) \\
-\frac{1}{2}\left(\frac{C_{5}}{C_{4}} x-\frac{C_{2} \Lambda_{2}}{C_{3} C_{4}} y+\frac{\Lambda_{3}}{C_{4}} x^{\prime}+\frac{C_{2} C_{5}}{C_{4}} y^{\prime}\right)
\end{array}\right]
$$

where

$$
\begin{aligned}
& C_{4}=C_{1} \Lambda_{3}^{2}+C_{2} \Lambda_{2}^{2}=\frac{\sqrt{\sigma_{i}\left(9 \sigma_{i}-8\right)}}{2} \\
& C_{5}=2 \sigma_{i}+1
\end{aligned}
$$

Consequently Eq. (4) is transformed into a new coordinate as

$$
Z^{\prime}=D Z+\tilde{B} \tilde{F}(T Z)
$$

where

$$
\tilde{\boldsymbol{B}}=\boldsymbol{T}^{-1} \boldsymbol{B}=\left[\begin{array}{ccc}
0 & 0 & 1 \\
0 & 0 & 0 \\
0 & C_{1} C_{5} / C_{4} & 0 \\
-\Lambda_{2} / C_{4} & 0 & 0 \\
\Lambda_{3} /\left(2 C_{4}\right) & -C_{2} C_{5} /\left(2 C_{4}\right) & 0 \\
-\Lambda_{3} /\left(2 C_{4}\right) & -C_{2} C_{5} /\left(2 C_{4}\right) & 0
\end{array}\right]
$$

Let $\boldsymbol{Z}=\left[z_{1}^{c}, z_{2}^{c}, z_{3}^{c}, z_{4}^{c}, z^{s}, z^{u}\right]^{T}=\left[\boldsymbol{z}^{c}, z^{s}, z^{u}\right]^{T}$ and $\boldsymbol{G}(\boldsymbol{Z})=$ $\tilde{B} \tilde{\boldsymbol{F}}(\boldsymbol{T Z})=[\boldsymbol{f}(\boldsymbol{Z}), g(\boldsymbol{Z}), h(\boldsymbol{Z})]^{T}\left(\boldsymbol{f}: \boldsymbol{R}^{4} \rightarrow \boldsymbol{R}^{4}, g, h: \boldsymbol{R}^{1} \rightarrow\right.$ $\boldsymbol{R}^{1}$ ), Eq. (6) can be divided to

$$
\begin{aligned}
& \boldsymbol{z}^{c \prime}=\boldsymbol{P} z^{c}+\boldsymbol{f}\left(z^{c}, z^{s}, z^{u}\right) \\
& z^{s \prime}=Q z^{s}+g\left(z^{c}, z^{s}, z^{u}\right) \\
& z^{u \prime}=R z^{u}+h\left(z^{c}, z^{s}, z^{u}\right)
\end{aligned}
$$

As a result, Eq. (4) can be transformed into the diagonal form to apply to the center manifold theory.

\subsection{Computing center manifold and reduced system}

The analytical approximation method proposed by Suzuki et al. ${ }^{11)}$ is applied to solve Eq. (7). According to the center manifold theory, ${ }^{13)}$, 14) when the state is in the vicinity of the origin, the system can be reduced to the system lying on the center manifold, and the local center manifold is expressed as the function not of time but of the state in the reduced system. That is, if the state $\boldsymbol{Z}$ is in a neighborhood of the origin, the reduced system of Eq. (7) and the center manifold can be expressed as

$$
\begin{aligned}
\boldsymbol{z}^{c \prime} & =\boldsymbol{P} z^{c}+\boldsymbol{f}\left(z^{c}, \phi\left(z^{c}\right), \psi\left(z^{c}\right)\right) \\
z^{s} & =\phi\left(z^{c}\right) \\
z^{u} & =\psi\left(z^{c}\right)
\end{aligned}
$$

This means that the state of stable and unstable components are constrained by the reduced system, and consequently the dimension of the dynamics is reduced from six to four. 
Let define the sequences $\left\{z_{k}^{c}(t, \boldsymbol{\xi})\right\},\left\{z_{k}^{s}\left(z_{k}^{c}(t, \boldsymbol{\xi})\right)\right\},\left\{z_{k}^{u}\left(z_{k}^{c}(t, \boldsymbol{\xi})\right)\right\}$, $\left\{z_{k}^{s}(\boldsymbol{\xi})\right\}$ and $\left\{z_{k}^{u}(\boldsymbol{\xi})\right\}$ as

$$
\begin{aligned}
& \boldsymbol{z}_{k+1}^{c}(t, \boldsymbol{\xi})=e^{\boldsymbol{P t}} \boldsymbol{\xi} \\
& \quad+\int_{0}^{t} e^{\boldsymbol{P}(t-\tau)} \boldsymbol{f}\left(z_{k}^{c}(\tau, \boldsymbol{\xi}), z_{k}^{s}\left(\boldsymbol{z}_{k}^{c}(\tau, \boldsymbol{\xi})\right), z_{k}^{u}\left(\boldsymbol{z}_{k}^{c}(\tau, \boldsymbol{\xi})\right)\right) d \tau \\
& z_{k+1}^{s}\left(\boldsymbol{z}_{k+1}^{c}(t, \boldsymbol{\xi})\right)=e^{Q t} z_{k+1}^{s}(\boldsymbol{\xi}) \\
& \quad+\int_{0}^{t} e^{Q(t-\tau)} g\left(\boldsymbol{z}_{k}^{c}(\tau, \boldsymbol{\xi}), z_{k}^{s}\left(\boldsymbol{z}_{k}^{c}(\tau, \boldsymbol{\xi})\right), z_{k}^{u}\left(\boldsymbol{z}_{k}^{c}(\tau, \boldsymbol{\xi})\right)\right) d \tau \\
& \quad+\int_{0}^{u} e^{R(t-\tau)} h\left(\boldsymbol{z}_{k}^{c}(\tau, \boldsymbol{\xi}), z_{k}^{s}\left(\boldsymbol{z}_{k}^{c}(\tau, \boldsymbol{\xi})\right), z_{k}^{u}\left(z_{k}^{c}(\tau, \boldsymbol{\xi})\right)\right) d \tau \\
& z_{k+1}^{s}(\boldsymbol{\xi})=\int_{-\infty}^{0} e^{-Q \tau} g\left(\boldsymbol{z}_{k}^{c}(\tau, \boldsymbol{\xi}), z_{k}^{s}\left(\boldsymbol{z}_{k}^{c}(\tau, \boldsymbol{\xi})\right), z_{k}^{u}\left(\boldsymbol{z}_{k}^{c}(\tau, \boldsymbol{\xi})\right)\right) d \tau \\
& z_{k+1}^{u}(\boldsymbol{\xi})=-\int_{0}^{\infty} e^{-R \tau} h\left(z_{k}^{c}(\tau, \boldsymbol{\xi}), z_{k}^{s}\left(z_{k}^{c}(\tau, \boldsymbol{\xi})\right), z_{k}^{u}\left(\boldsymbol{z}_{k}^{c}(\tau, \boldsymbol{\xi})\right)\right) d \tau \\
& \quad k=0,1,2, \cdots \quad(9)
\end{aligned}
$$

where $\boldsymbol{\xi}=\left[\xi_{1}, \xi_{2}, \xi_{3}, \xi_{4}\right]^{T}$ is the initial state of $\boldsymbol{z}_{k}^{c}$, and initial conditions are set as

$$
\begin{array}{ll}
\boldsymbol{z}_{0}^{c}(t, \boldsymbol{\xi})=e^{\boldsymbol{P} t} \boldsymbol{\xi}, & z_{0}^{s}\left(\boldsymbol{z}_{0}^{c}(t, \boldsymbol{\xi})\right)=0, \\
z_{0}^{s}(\boldsymbol{\xi})=0, & z_{0}^{u}(\boldsymbol{\xi})=0
\end{array}
$$

According to 11$)$, the sequences $\left\{\boldsymbol{z}_{k}^{c}(t, \boldsymbol{\xi})\right\},\left\{z_{k}^{s}\left(\boldsymbol{z}_{k}^{c}(t, \boldsymbol{\xi})\right)\right\}$ and $\left\{z_{k}^{u}\left(z_{k}^{c}(t, \xi)\right)\right\}$ are the approximating solutions to the exact solutions of Eq. (7) on the center manifold. In addition, these sequences are uniformly convergent to the exact solutions, $\left\{\boldsymbol{z}^{c}(t, \boldsymbol{\xi})\right\},\left\{z^{s}\left(\boldsymbol{z}^{c}(t, \boldsymbol{\xi})\right)\right\},\left\{z^{u}\left(\boldsymbol{z}^{c}(t, \boldsymbol{\xi})\right)\right\}$, respectively, as $k \rightarrow \infty$.

By applying Eq. (9) to the divided system Eq. (7), the approximating solutions can be computed. The solutions are expressed in the new coordinate system. Therefore we can obtain the solutions in the original system by multiplying the transformation matrix $\boldsymbol{T}$.

Though the differential correction method is a powerful method to obtain periodic orbits, whole initial conditions must be determined. On the other hand, the most remarkable feature of the proposed method is that when the four initial values of $\boldsymbol{\xi}$ is determined, the rest are specified. Thus, it is possible to determine unstable periodic orbits along the center manifold by applying an arbitrary initial value $\boldsymbol{\xi}$.

\section{Simulation for Sun-Earth CRTBP}

In this section, the Sun-Earth CRTBP around $L_{2}$ is considered to demonstrate the effectiveness of the proposed method. The non-dimensional parameters around the $L_{2}$ are given as: $\rho=$ $3.054248396 \times 10^{-6}, l_{2}=1.010091760$ and $\sigma_{2}=3.939308152$. First, the verification of the proposed method is discussed. Then the dependence between the initial condition and the obtained orbits is investigated. The simulation conditions are set as: non-dimensional time step is $1.0 \times 10^{-6}$ and time span is 10.0 (corresponding to about 580 days). When $\mid z_{k}^{s}\left(z_{k}^{c}(0, \xi)\right)-$ $z_{k+1}^{s}\left(z_{k+1}^{c}(0, \xi)\right) \mid<10^{-19}$ and $\left|z_{k}^{u}\left(z_{k}^{c}(0, \xi)\right)-z_{k+1}^{u}\left(z_{k+1}^{c}(0, \xi)\right)\right|<$ $10^{-19}$, the approximate solution is considered to be converged to the exact solution, and then the iterative operation is terminated. Each iteration step takes on average 1.0 second on a Intel Core
i7-4800MQ, 2.70 GHz, 16.0 GB RAM, notebook computer implementing by MATLAB R2013a. For easy understanding, the dimensional units are used in the following simulation results.

\subsection{The verification of the proposed method}

Firstly, the initial state $\boldsymbol{X}_{0}$ obtained by the differential correction method is used to verify the proposed method. This approach requires only the initial state $\xi$. The other initial values of stable and unstable components are determined successively. Therefore if the initial value $\boldsymbol{\xi}$ is equal to the first to fourth elements of $\boldsymbol{Z}_{0}=\boldsymbol{T}^{-1} \boldsymbol{X}_{0}$, the rest of initial values must be converged to the fifth and sixth elements of $\boldsymbol{Z}_{0}$ and the orbit obtained by the proposed method must correspond with the reference orbit. Now the initial value $\boldsymbol{X}_{0}$ of the reference halo orbit in the original coordinate is given as

$$
\boldsymbol{X}_{0}=\left[\begin{array}{c}
-2.255565294 \\
0 \\
2.227854183 \\
0 \\
11.80000000 \\
0
\end{array}\right] \times 10^{-3}
$$

Then it is transformed into the diagonal form $\boldsymbol{Z}_{0}$ as

$$
\boldsymbol{Z}_{0}=\boldsymbol{T}^{-1} \boldsymbol{X}_{0}=\left[\begin{array}{c}
0 \\
-4.422407468 \\
11.02194518 \\
0 \\
0.3890274115 \\
0.3890274115
\end{array}\right] \times 10^{-3}
$$

To verify the proposed method, the first to fourth elements of $\boldsymbol{Z}_{0}$ are set as the initial condition of $\boldsymbol{\xi}$.

The orbit obtained after 38 iteration is shown in Fig. 1. The red line is the reference halo orbit, the blue line is approximate solution and the blue point is the initial position obtained by the proposed method.

It can be seen from Fig. 1 that the reference and obtained orbits are overlapped. The approximate initial values $\left[\boldsymbol{z}^{c}(0, \boldsymbol{\xi}), z^{s}\left(\boldsymbol{z}^{c}(0, \boldsymbol{\xi})\right), z^{u}\left(\boldsymbol{z}^{c}(0, \boldsymbol{\xi})\right)\right]$, and thus $\left[\boldsymbol{\xi}, z^{s}(\boldsymbol{\xi}), z^{u}(\boldsymbol{\xi})\right]$ converged to the reference initial condition $Z_{0}$ as $k$ increases. Therefore by choosing the four initial values appropriately, the proposed method can compute the rest of parameters which configure a halo orbit.

Next, arbitrary four elements of $\boldsymbol{\xi}$ are used to generate a orbit on the center manifold. As a example, the initial value $\xi$ is set as $[1.0,-3.0,5.0,1.0]^{T} \times 10^{-3}$, where it is chosen to be small enough to satisfy the assumption of the center manifold theory. In Fig. 2, the quasi-periodic orbit is obtained after 21 iteration. This signifies that the proposed method restricts the motion on the center manifold and generates a bounded orbit by arbitrary four elements of $\boldsymbol{\xi}$.

\subsection{Dependence of initial condition and type of orbit}

In the previous subsection, the periodic and quasi-periodic orbits are determined by the proposed approach. In computing these orbits, only the initial values of the center manifold are needed, hence it is obvious that the initial value $\boldsymbol{\xi}$ determines the shape of resulting orbits. In this subsection, the relation between the initial value $\xi$ and the shape of the orbit is investigated. 


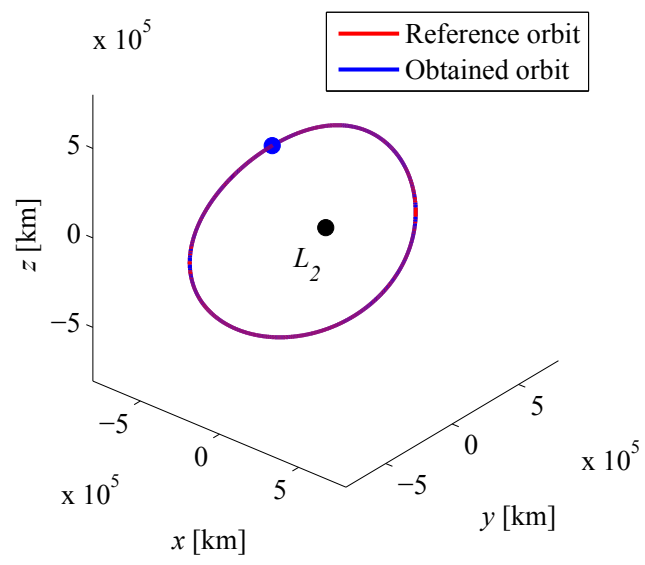

(a) 3-D view of trajecotry

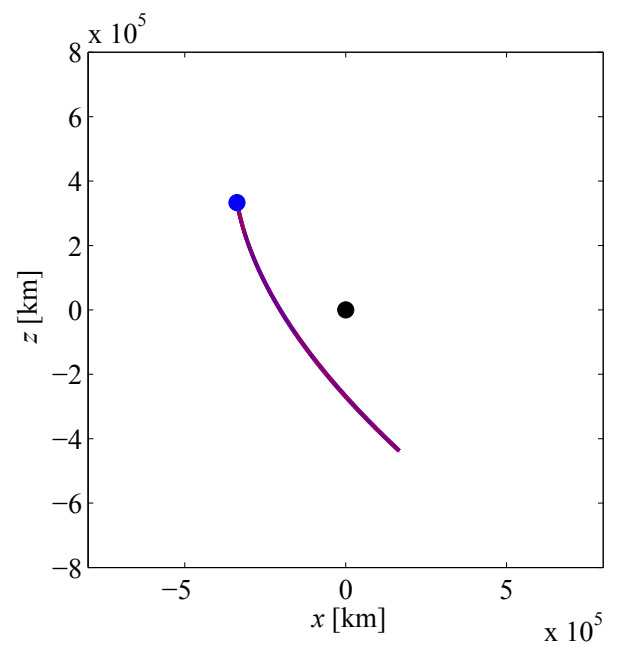

(c) projection onto the $x-z$ plane

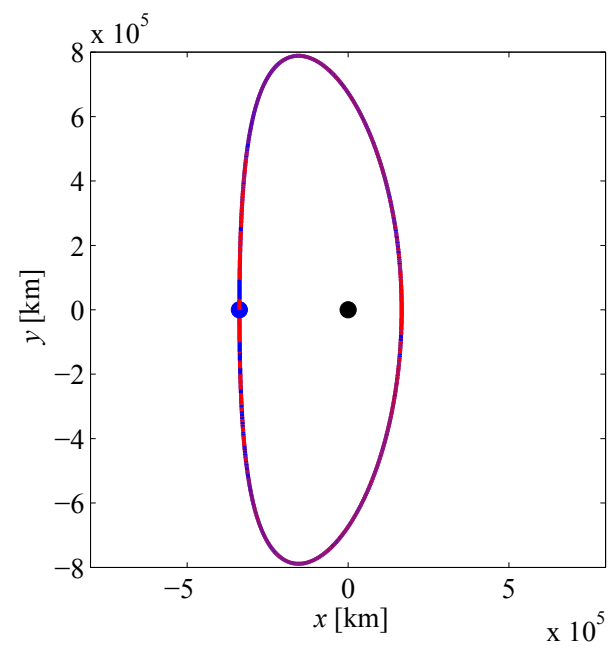

(b) projection onto the $x-y$ plane

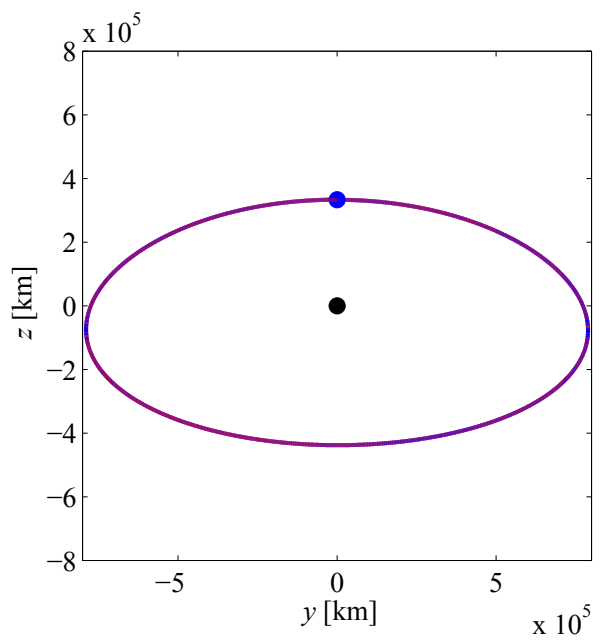

(d) projection onto the $y-z$ plane

Fig. 1. Halo orbit: Red and blue orbits are reference halo orbit and obtained by the proposed method, respectively. Blue point is the initial position.

To reveal the effect of the each element of $\boldsymbol{\xi}$, one element is set to $1.0 \times 10^{-3}$ and the rests are set to zero. The orbits obtained by each initial value are shown in the projection onto $x-y$ and $x-z$ plane in Fig. 3 - Fig. 6 .

All orbits obtained after 10 iteration is periodic or quasiperiodic in Fig. 3 - Fig. 6. When $\xi_{1}$ or $\xi_{2}$ is nonzero values, the orbits remain around the Lagrangian point and form three-dimensional quasi-periodic orbits whose amplitudes in the $z$-direction are much larger than those in the $x$ and $y$ directions (Figs. 3 and 4 ). In contrast, $\xi_{3}$ and $\xi_{4}$ have only effect on the in-plane motion (Figs. 5 and 6). In addition, the orbits by using only $\xi_{3}$ and $\xi_{4}$ are Lyapunov orbits. These are understandable from the following. The initial condition $\boldsymbol{X}_{0}$ obtained by the proposed method is expressed as

$$
\boldsymbol{X}_{0}=\left[\begin{array}{c}
-C_{2} \xi_{3}-2 C_{1} z^{s}(\boldsymbol{\xi}) \\
-C_{3} L_{3} \xi_{4} \\
-\xi_{2} / L_{1} \\
-C_{2} L_{2} \xi_{4} \\
\xi_{3}+2 z^{s}(\boldsymbol{\xi}) \\
\xi_{1}
\end{array}\right]
$$

where $z^{s}(\boldsymbol{\xi})=z^{u}(\boldsymbol{\xi})$ is used. In the case of Figs. 3 and 4, Eq. (10) becomes

$$
\boldsymbol{X}_{0}=\left[\begin{array}{c}
-2 C_{1} z^{s}(\boldsymbol{\xi}) \\
0 \\
-\xi_{2} / L_{1} \\
0 \\
2 z^{s}(\boldsymbol{\xi}) \\
\xi_{1}
\end{array}\right]
$$

From the center manifold theory, in the vicinity of the equilibrium points, the orders of $z^{s}$ and $z^{u}$ are sufficiently smaller than that of $z_{i}^{c}(i=1,2,3,4)$. In addition, in the Sun-Earth $L_{2}$ system, the constants are $C_{1}=0.7382209754$ and $L_{1}=1.985052479$. Therefore the third and sixth components of Eq. (11) which affect the $z$-direction motion are larger than the first and fifth ones. This is because the out-of-plane components are much bigger than in-plane ones in Figs. 3 and 4. On the other hand, the reason why the Figs. 5 and 6 cases are Lyapunov orbits is obvious from Eq. (10) that the $z$-direction terms are zero. Therefore it can be said that when a Lyapunov orbit is designed, it is necessary to give nonzero values to $\xi_{3}$ or $\xi_{4}$ and $\xi_{1}$ and $\xi_{2}$ should be zero. Conversely, in the case of designing three-dimensional periodic or quasi-periodic orbits like halo or Lissajous orbits, at least either $\xi_{1}$ or $\xi_{2}$ requires nonzero values. 


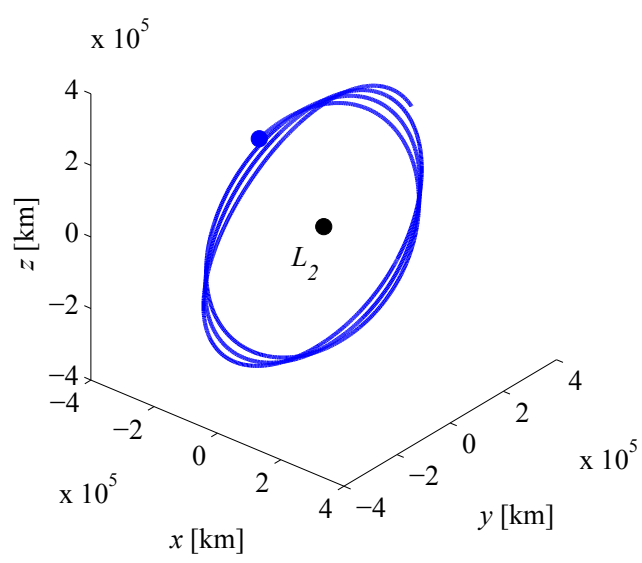

(a) 3-D view of trajecotry

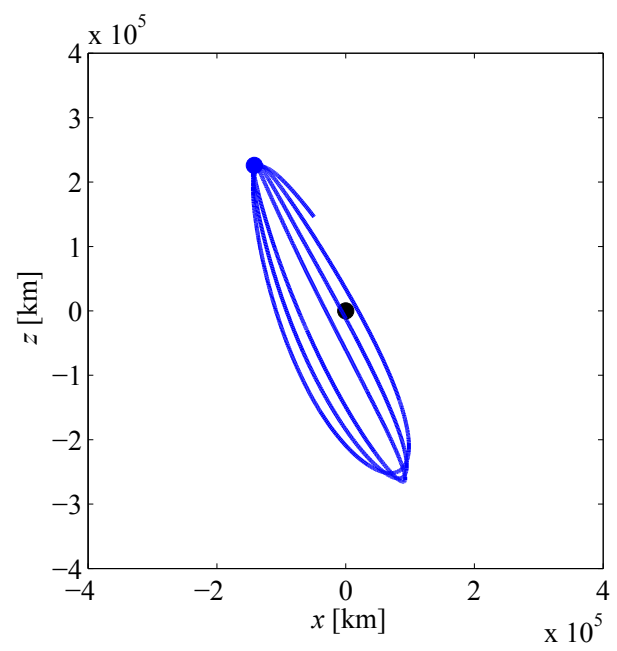

(c) projection onto the $x-z$ plane

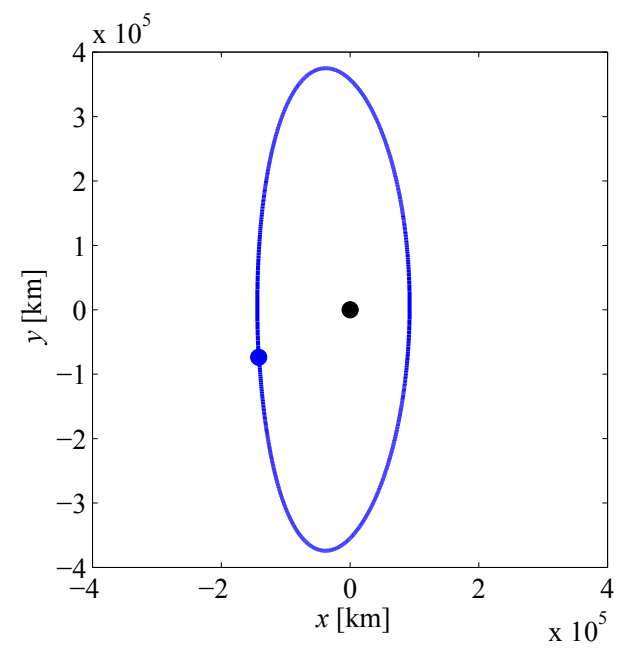

(b) projection onto the $x-y$ plane

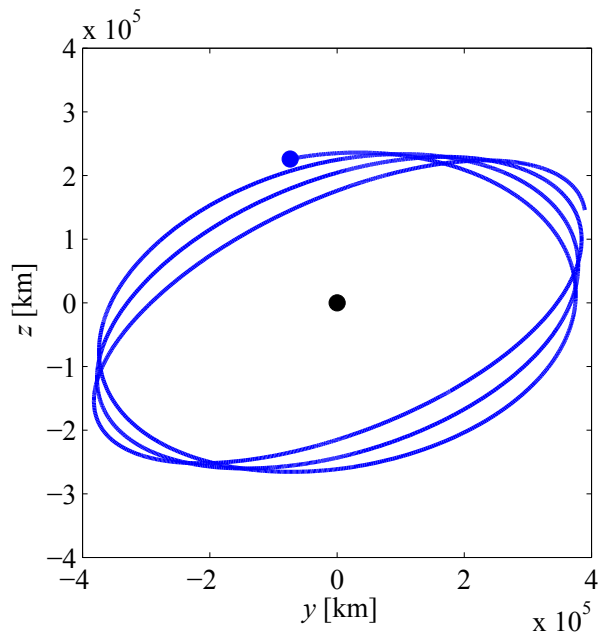

(d) projection onto the $y-z$ plane

Fig. 2. Quasi-periodic orbit (Lissajous orbit).

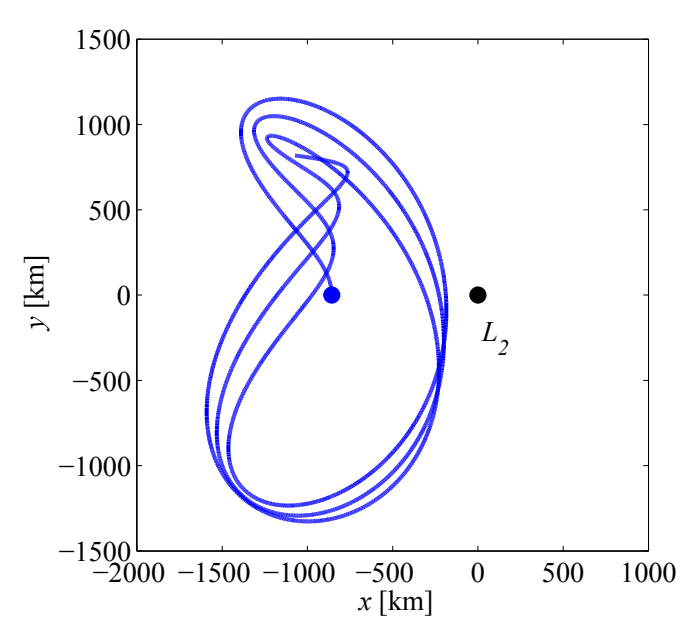

(a) projection onto the $x-y$ plane

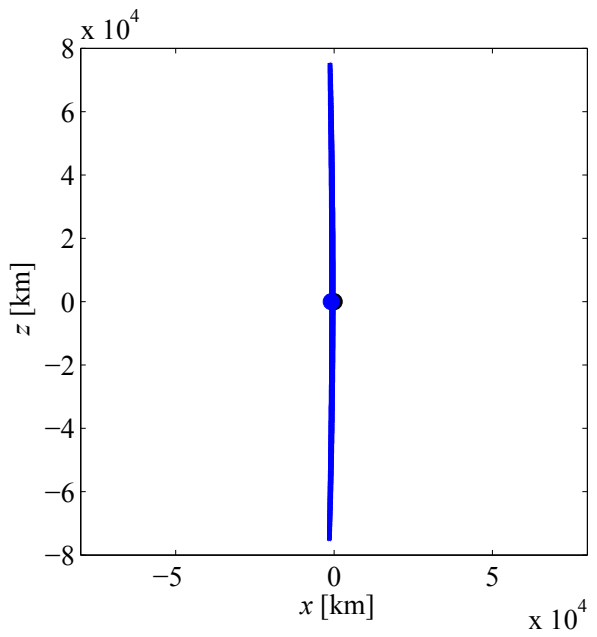

(b) projection onto the $x-z$ plane

Fig. 3. Quasi-periodic orbit: $\xi=[1,0,0,0]^{T} \times 10^{-3}$.

Note that the proposed method computes the fourdimensional center manifold in the vicinity of the Lagrangian point in the CRTBP. Hence it successively provides a bounded orbit by using an arbitrary initial condition, $\boldsymbol{\xi}$. However there are mainly two difficulties. The first problem is that the pro- posed method cannot distinguish whether it be the initial condition of a quasi-periodic orbit or that of a periodic orbit before the calculation. Whereas a quasi-periodic orbit has two different periods in the in-plane and out-of-plane directions, a periodic orbit is formed as a special case when both periods are equal. 


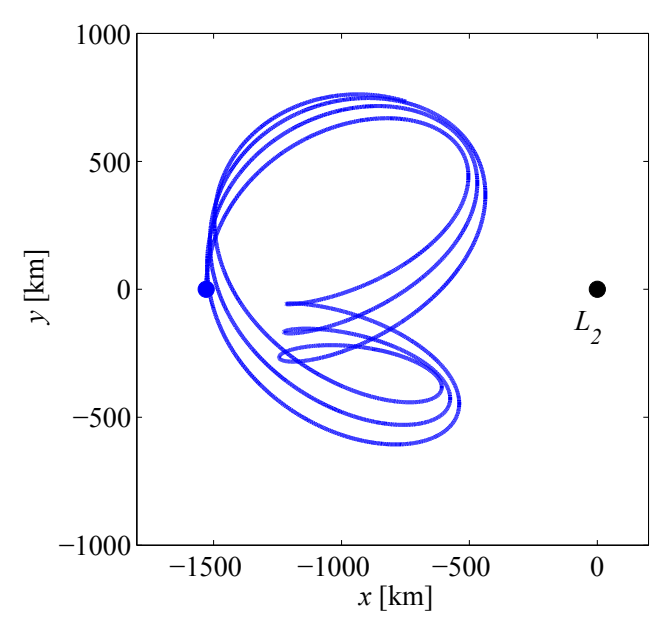

(a) projection onto the $x$ - $y$ plane

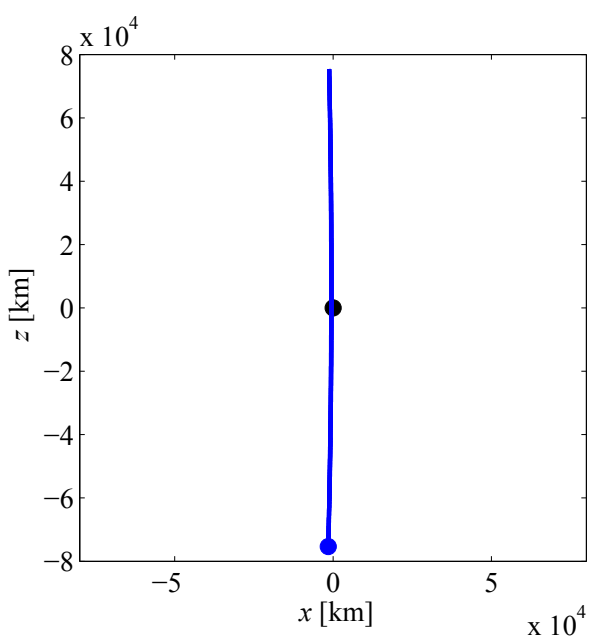

(b) projection onto the $x-z$ plane

Fig. 4. Quasi-periodic orbit: $\xi=[0,1,0,0]^{T} \times 10^{-3}$.

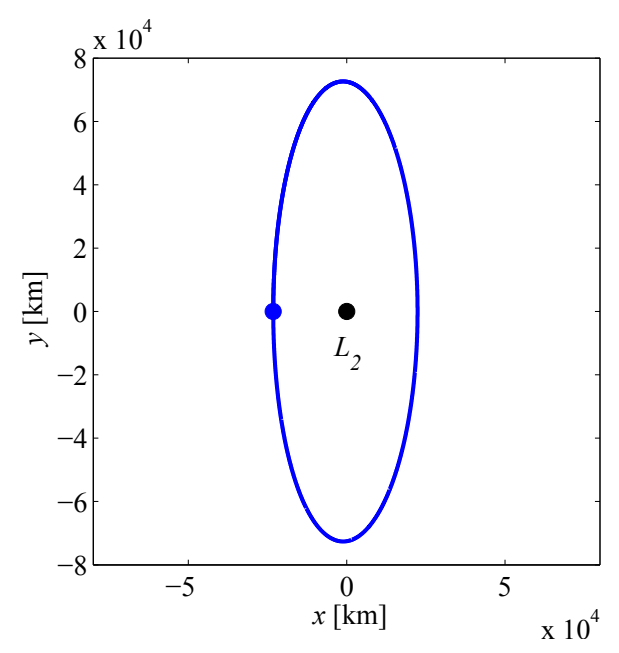

(a) projection onto the $x-y$ plane

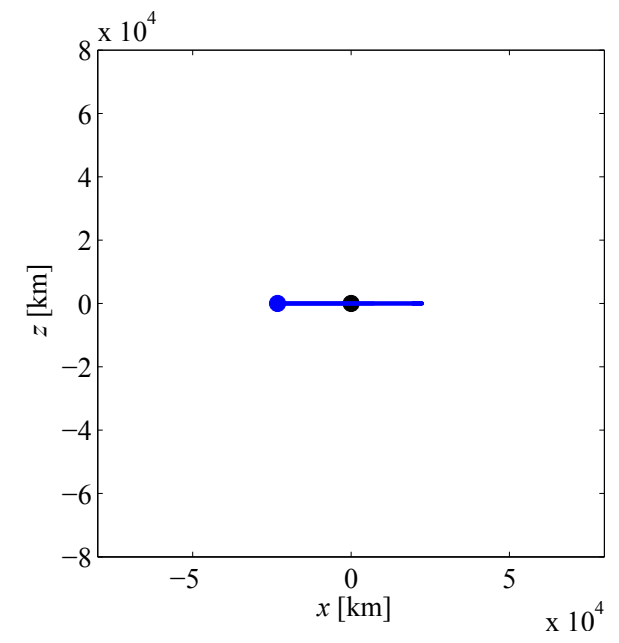

(b) projection onto the $x-z$ plane

Fig. 5. Periodic orbit (Lyapunov orbit): $\xi=[0,0,1,0]^{T} \times 10^{-3}$.

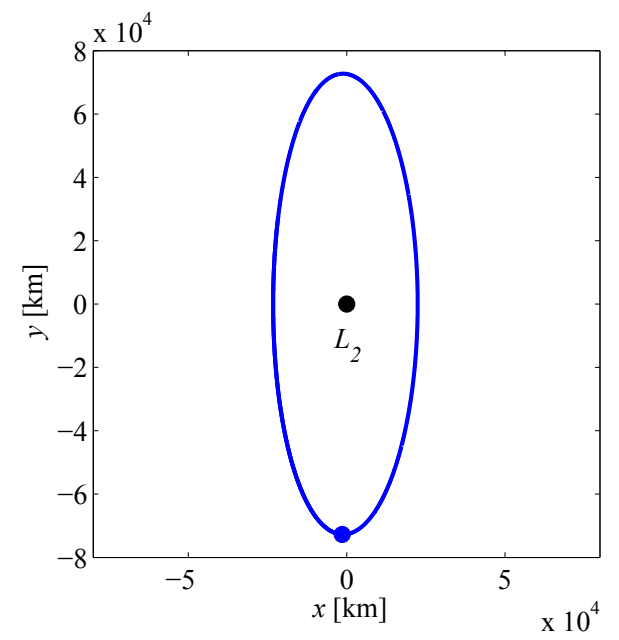

(a) projection onto the $x-y$ plane

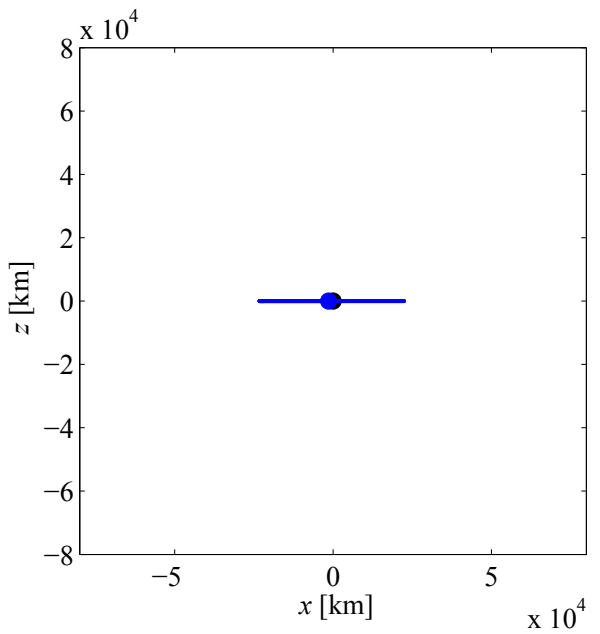

(b) projection onto the $x-z$ plane

Fig. 6. Periodic orbit (Lyapunov orbit): $\xi=[0,0,0,1]^{T} \times 10^{-3}$.

A periodic orbit is obtained only if the initial condition is sufficiently close to one of the periodic orbit. Otherwise, as shown in Figs. 3 and 4, a quasi-periodic orbit is obtained. Therefore to obtain a periodic orbit, an initial condition $\boldsymbol{\xi}$ must be corrected.
The other difficulty is that the shape of the obtained orbit is difficult to predict before the calculation. This is because the transformed variables $z^{c}$ are mingled with the components of the positions and velocities. However the geometry of the orbit 
obtained by an arbitrary initial condition $\xi$ can be continuously altered by slightly changing the initial condition. Repeating the adjustment of the initial condition of the in-plane and out-ofplane directions respectively, the desired orbit can be obtained.

\section{Conclusion}

A new approach based on the center manifold theory has been proposed to design periodic and quasi-periodic orbits in the vicinity of the Lagrangian points in the circular restricted three-body problem. The proposed method is semi-analytical unlike the differential correction method and the LindstedtPoincaré method. First, to verify the proposed method, it has been confirmed that it is possible to design the periodic or quasiperiodic orbits by using arbitrary four initial values. Next, the effect of the each element of the four initial values has been investigated. It has been revealed that two of four have effect on the in-plane motion, and the rest two do on the out-of-plane motion. Therefore by adjusting each element of the initial values, we can obtain the desired orbits.

\section{References}

1) Prussing, J. E. and Conway, B. A.: Orbital Mechanics, (2nd Edition), Oxford University Press, 2013.

2) Kinoshita, H.: Celestial Mechanics and Orbital Dynamics, Tokyo University Press, 1998 (in Japanese).

3) Howell, K. C.: Three-Dimensional Periodic 'Halo' Orbits, Celestial Mechanics, 32-1 (1984), pp. 53-71.

4) Le Bihan, B., Kokou, P. and Lizy-Destrez, S.: Computing an Optimized Trajectory between Earth and an EML2 Halo Orbit, AIAA Scitech, AIAA 2014-0450, 2014.

5) Kolemen, E., Kasdin, N. J., and Gurfil, P.: Quasi-Periodic Orbits of the Restricted Three-Body Problem Made Easy, New Trends in Astrodynamics and Applications III (AIP Conference Proceedings Volume 886), 886 (2007), pp. 68-77.

6) Folta, D., Pavlak, T., Haapala, A., and Howell, K.: Preliminary Design Considerations for Access and Operations in Earth-Moon L1/L2 Orbits, 23rd AAS/AIAA Space Flight Mechanics Meeting, (2013), pp. 10-14.

7) NASA Jet Propulsion Laboratory GENESIS Search for Origin, http://www.jpl.nasa.gov/ (cited 15 January 2015).

8) Howell, K. C. and Pernicka, H. J.: Numerical Determination of Lissajous Trajectories in the Restricted Three-Body Problem, Celestial Mechanics, 41 (1988), pp. 107-124.

9) Richardson, D. L.: Analytical Construction of Periodic Orbits about the Collinear Points, Celestial Mechanics, 22 (1980), pp. 241-253.

10) Gomez, G., Masdemont, J. and Mondelo, J. M.: Libration Point Orbits: a Survey from the Dynamical Point of View, Libration point orbits and applications, Proceedings of the Conference Aiguablava, Spain, (2002), pp. 10-14.

11) Suzuki, H., Sakamoto, N. and Celikovsky, S.: Analytical Approximation Method for the Center Manifold in the Nonlinear Output Regulation Problem, 47th IEEE Conference on Decision and Control, (2008), pp. 1163-1168.

12) Wie, B.: Space Vehicle Dynamics and Control, (2nd Edition), AIAA, 2008.

13) Khalil, H. K.: Nonlinear Systems, (3rd Edition), Prentice Hall, 2002.

14) Perko, L.: Differential Equations and Dynamical Systems, (3rd Edition), Springer, 2001. 